\title{
IT-Architektur
}

\section{Matthias Knoll}

(C) Springer Fachmedien Wiesbaden GmbH, ein Teil von Springer Nature 2018

Häufig wird heute von ,bimodaler IT“ gesprochen. Denn in vielen Unternehmen existieren (noch) zwei „Welten“ parallel: Einerseits sind dies die verlässlichen, stabilen IT-Lösungen, die seit nunmehr etwa 60 Jahren das Geschäft unterstützen, dessen Kernkompetenzen im Nicht-IT-Bereich liegen (IT als „Factory“). Andererseits sind es neue Anwendungssysteme und vor allem Plattformen, die als „Strategische Waffe" neuartige Produkte, Dienstleistungen und Geschäftsmodelle ermöglichen. Die Kernkompetenzen sind dort unmittelbar in der und durch die IT abgebildet. Gleichzeitig lässt sich im Rahmen der digitalen Transformation von Unternehmen aller Branchen ein immer schnelleres Zusammenwachsen der beiden „Welten“ beobachten.

Eng mit einer solchen Entwicklung verbunden ist daher drängender denn je die Frage nach der ,richtigen“ IT-Architektur im Sinne einer möglichst langlebigen und flexiblen Lösung, die den sich rasch verändernden fachlichen Anforderungen folgen kann.

Dabei werden unter Architektur im Kontext von Informationssystemen ein strukturierendes Element, eine modellhafte Beschreibung der grundsätzlichen Struktur aller Elemente eines Systems sowie deren Beziehungen zueinander verstanden. Für ein gutes Business-IT-Alignment (siehe hierzu auch das von Stefan Reinheimer und Susanne Robra-Bissantz in der Edition HMD herausgegebene Buch „BusinessIT-Alignment") geht diese modellhafte Beschreibung über technische Sachverhalte hinaus und mündet im Begriff der Unternehmensarchitektur. Aus eher implementierungsnaher Sicht beschreibt der Begriff die Struktur informationstechnischer Systeme, ihre Zusammensetzung aus verschiedenen (Code-)Elementen und deren

M. Knoll (ه)

Hochschule Darmstadt, Haardtring 100, 64295 Darmstadt, Deutschland

E-Mail: matthias.knoll@h-da.de 
- auch Anwendungs-übergreifendes - Zusammenwirken. Ein Beispiel hierfür sind Software-Architekturen.

Doch was in der Theorie einfach und logisch klingt, kann in der Praxis schwer umsetzbar sein.

Mitunter wurde und wird die Architekturfrage in Unternehmen überhaupt nicht gestellt, sondern einfach mit der Entwicklung begonnen, weil das Wissen und/oder die Zeit für weitergehende Überlegungen fehlen oder Unklarheit bezüglich der ITStrategie besteht (zu diesem Aspekt sei ergänzend auf die von Susanne Strahringer und Thomas Widjaja herausgegebene HMD-Ausgabe 54/3, Juni 2017, verwiesen). Mitunter wurde und wird aber auch zu ausführlich darüber diskutiert, welcher Weg der richtige sei, was letztlich eine Nutzung stark verzögert oder obsolet werden lässt, weil sich neue Ansätze schneller durchsetzen, als Entscheidungen getroffen werden. Doch selbst wenn - in der Mehrzahl der Fälle - in akzeptabler Zeit eine technisch, fachlich und kostenseitig tragbare Lösung gefunden wird, die dann auch erfolgreich und mit gebotener Sorgfalt umgesetzt wird, sind die Verantwortlichen in den ITAbteilungen vielfach von der Realität eingeholt worden.

Denn drei wesentliche Hindernisse können die Frage der Langlebigkeit nahezu unlösbar machen:

1. Die unaufhaltsame technologische Entwicklung, die morgen Konzepte ermöglicht, die heute undenkbar sind und daher in Überlegungen auch nicht antizipiert werden können.

2. Zeit- und Kostendruck, der teilweise eigenverschuldet oder durch (vermeintlichen) Marktdruck die Umsetzung von guten Lösungen behindert. Fachliche Anforderungen müssen rasch umgesetzt werden, doch die bislang genutzte IT-Architektur setzt Grenzen. Die Folge: Es werden neuere Ansätze mit alten Ansätzen verbunden - mit allen technischen und semantischen Problemen.

3. Fehlendes strategisches Denken, ein Defizit, das vielfach durch einen in der jeweilige Unternehmenskultur verankerten kurzfristig orientierten Planungs- und Entscheidungshorizont entstanden ist und sich nicht selten auch auf die Rahmenbedingungen für die Weiterbildung des Personals erstreckt.

So entstand eine mehr oder weniger gut durchschaubare, eine sogenannte ,historisch gewachsene" IT-Landschaft, in der unterschiedlichste Architektur- und Technologie-Ansätze nebeneinander bestehen mussten.

Seit einigen Jahren nun fordert die digitale Transformation der Unternehmen, das Thema IT-Architektur konzentriert zu betrachten. Denn nicht nur subjektiv, sondern objektiv in zunehmend schnellerer Folge müssen von Business-Seite initiierte neue Ideen technisch umgesetzt werden, gleichzeitig konkurrieren viele neue Technologien mit ihren darunter liegenden Referenzmodellen und Architekturmustern um die Gunst der Entwickler. Lebenszyklen aller involvierten Elemente verkürzen sich. Die ohnehin schon historisch gewachsene Landschaft droht nun in noch kürzerer Zeit noch unübersichtlicher zu werden. Das hat gravierende Folgen - nicht zuletzt aus Risikogesichtspunkten. Vielleicht ist es nur ein subjektiver Eindruck der betroffenen IT-Sicherheitsexperten, doch scheinen Sicherheitsprobleme aufgrund fehlerhafter Hard- und Software-Konzeption zuzunehmen. Besonders dramatisch wird dies für alle Unternehmen, die zur kritischen Infrastruktur gezählt werden, aber auch für 
„normale“ Produktionsunternehmen, deren Fertigung von Angreifern kompromittiert werden kann oder gar für Unternehmen, deren Geschäftsmodelle zu einhundert Prozent von der IT abhängen.

Entsprechend sorgfältig sollte der Blick auf das Thema IT-Architektur sein. Denn offensichtlich unterstützt eine, gute“ IT-Architektur bei der systematischen Umsetzung immer neuer Anforderungen und der Beherrschung von Risiken auch und gerade in Zeiten des raschen Wandels.

Die vorliegende Ausgabe der HMD zum Thema IT-Architektur gibt im Einführungsbeitrag und sieben weiteren Schwerpunktbeiträgen Impulse für die Arbeit mit dem Themenkomplex IT-Architektur. Allen Beiträgen gemeinsam ist die Erkenntnis, dass eine Architektur einen wichtigen Erfolgsfaktor in der Komplexitätsreduktion moderner IT darstellt. Der Einführungsbeitrag verweist zudem auf die hohe Bedeutung von Referenzarchitekturen. Sie könnten die Entwicklung mithilfe von Standards oder konkretem Architektur- und/oder Domänenwissen erleichtern. Mit der im Beitrag vorgestellten Systematik und der Diskussion des Begriffes lassen sich einschlägige Ansätze einordnen und die darin enthaltenen Überlegungen hinsichtlich einer Übertragbarkeit auf das eigene Unternehmen evaluieren. Ein weiterer Beitrag thematisiert explizit die Notwendigkeit einer Strukturierung im Rahmen der Digitalisierung und benennt im „Enterprise Architecture Digital Readiness Framework“ wichtige Einflussfaktoren. An ihnen entlang werden mögliche Lösungsansätze in den Bereichen Kundenschnittstelle, Qualifizierung, Optimierung des operativen Architekturmanagements und einer notwendigen Feedbackkultur aufgezeigt.

Für Entscheider mit Kostenverantwortung interessant sein dürfte auch die Betrachtung von Kosten im Kontext der Optimierung bestehender Unternehmensarchitekturen. Der Beitrag berücksichtigt dabei nicht nur den Ist-Zustand, sondern auch Transitionskosten, die vom Ist-Zustand zum optimalen Zustand aufgewendet werden müssen, und das im Zusammenspiel mit Betriebskosten.

Ebenso beleuchtet wird der Einsatz von IT-Architekturen in unterschiedlichen Sektoren, beispielhaft in Produktionsunternehmen und Krankenhäusern. Beide Beiträge zeigen anhand konkreter Gestaltungsempfehlungen, dass es nicht nur sinnvoll, sondern vielfach zwingend notwendig ist, sich an Architekturen zu orientieren. Dass auch bei der Entwicklung von Städten der Architekturgedanke nicht nur aus baulicher Sicht relevant sein kann, zeigt ein Beitrag über eine Architektur zur Transformation von Städten in Human Smart Cities. Weiterhin wird - entsprechend der Aktualität der Problematik - ein Modell zur Sicherstellung der Anforderungen aus der DSGVO und den damit verbundenen vielfältigen Herausforderungen unter Zuhilfenahme eines passend gestalteten und etablierten Unternehmensarchitekturmanagements (EAM) vorgestellt. Und schließlich rundet eine Betrachtung der Architekturfrage aus Sicht des IT-Risikomanagements und der IT-Prüfung das Themenfeld ab. Der Beitrag zeigt am Three-Lines-of-Defence-Modell auf, welche Herausforderungen auf die einzelnen „Verteidigungslinien“ zukommen, wo welche Verantwortung angesiedelt sein sollte und welche Aufgaben zu übernehmen sind.

Vier weitere Beiträge im Spektrum zu Leitlinien für das menschliche Handeln in einer digitalisierten Welt, zu Arbeit 4.0, zur Implementierung des Kreditkartenstandards PCI-DSS sowie zu einem Bewertungsverfahren für Anreize zum Teilen von Daten für digitale Geschäftsmodelle runden die Ausgabe ab. 
Die beiden Rezensionen werfen einen Blick auf Literatur zum Schwerpunkt. Besprochen wird ein „Klassiker“ zum Thema IT-Unternehmensarchitektur (mit ebendiesem Titel), der mittlerweile in der 3. Auflage vorliegt, sowie ein Buch, das sich mit der operativen Umsetzung von Architektur-Überlegungen beschäftigt (Langlebige Software-Architekturen). Beide Bücher bieten gute Hilfestellungen für all diejenigen, die zukunftsfähige IT-Strategien auf Basis durchdachter, langlebiger Architekturkonzepte in Anwendungen überführen.

Mein herzlicher Dank gilt allen Autorinnen und Autoren dieser Ausgabe, die ihr Wissen mit uns teilen und so wertvolle Impulse für die Diskussion geben.

Ich wünsche Ihnen eine spannende Lektüre, aus der Sie viel Wissen mitnehmen können und freue mich über Ihr Lob, Ihre Anregungen und Ihre Kritik.

Matthias Knoll 